\title{
Rhomboid proteins: conserved membrane proteases with divergent biological functions
}

\author{
Sinisa Urban ${ }^{1}$ \\ Department of Molecular Biology and Genetics, Johns Hopkins University School of Medicine, \\ Baltimore, Maryland 21205, USA
}

\begin{abstract}
The rhomboid gene was discovered in Drosophila, where it encodes a seven transmembrane protein that is the signal-generating component of epidermal growth factor (EGF) receptor signaling during development. Although metazoan developmental regulators are rarely conserved outside the animal kingdom, rhomboid proteins are conserved in all kingdoms of life, but the significance of this remains unclear. Recent biochemical reconstitution and high-resolution crystal structures have provided proof that rhomboid proteins function as novel intramembrane proteases, with a serine proteaselike catalytic apparatus embedded within the membrane bilayer, buried in a hydrophilic cavity formed by a protein ring. A thorough consideration of all known examples of rhomboid function suggests that, despite biochemical similarity in mechanism and specificity, rhomboid proteins function in diverse processes including quorum sensing in bacteria, mitochondrial membrane fusion, apoptosis, and stem cell differentiation in eukaryotes; rhomboid proteins are also now starting to be linked to human disease, including early-onset blindness, diabetes, and parasitic diseases. Regulating cell signaling is at the heart of rhomboid protein function in many, but not all, of these processes. Further study of these novel enzymes promises to reveal the evolutionary path of rhomboid protein function, which could provide insights into the forces that drive the molecular evolution of regulatory mechanisms.
\end{abstract}

Developmental biology has progressed from descriptive observation to mechanistic analysis in the latter half of the 20th century (Wolpert 1998). Powerful genetic tools applied to model organisms have led to an intricate and beautiful picture of how genes build complex organisms. Parallel analyses in diverse animals and sequencing of

[Keywords: Regulated intramembrane proteolysis; cell signalling; presenilin; malaria; Toxoplasma; Plasmodium]

${ }^{1}$ Correspondence.

E-MAIL surban@jhmi.edu; FAX (410) 502-3178.

Article is online at http://www.genesdev.org/cgi/doi/10.1101/gad.1488606. their genomes revealed that a relatively small number of master genes and core regulatory pathways are used repeatedly in different contexts to build complex patterns, and these factors are conserved among animals.

In addition to revealing some of the underlying logic of how organisms are constructed, these approaches were powerful in identifying the factors that mediate this intricate level of organization. But little is still known about how these key regulators exert their functions biochemically, which is central to understanding core biological processes that drive development at the molecular level.

Most developmental regulators are conserved in the animal kingdom but are not found in other kingdoms, including plants, perhaps because plants and animals evolved multicellular development after splitting from a common ancestor (Meyerowitz 2002). But in recent years a small number of factors have been discovered that are conserved across kingdoms, raising the exciting possibility that they are key regulators that evolved very early. One such class of regulatory proteins are intramembrane proteases.

Intramembrane proteolysis, the cleavage of membrane proteins within their membrane spanning segments, has recently been discovered to be a new regulatory paradigm through the study of completely unrelated biological and pathological processes (Brown et al. 2000; Steiner and Haass 2001; Urban and Freeman 2002; Weihofen and Martoglio 2003; Wolfe and Kopan 2004). Although three types of proteases are known to catalyze intramembrane proteolysis, and this field has seen exciting new advances, my aim is to discuss rhomboid proteins alone, and consider their functions as a family. These enzymes were originally identified by classical developmental genetics, but recent work has now uncovered roles for rhomboid proteins in unrelated biological processes, some of which have implications for human health. Although the majority of their known functions appear to be in regulating cell signaling in diverse organisms, implication of rhomboid proteins in unrelated processes promises to help reveal the evolutionary path of this central mode of regulation. 


\section{Rhomboid proteins: the basics}

\section{A short history of a classical developmental fly gene}

In what could be described as the first animal genomics project, screens were initiated in the 1970s to identify all genes required for Drosophila embryonic patterning (Jurgens et al. 1984; Nusslein-Volhard et al. 1984). The importance of these saturation screens to biology are remarkable, even in retrospect. Infiltration of diverse fields by the unique and memorable Drosophila names, such as patched in cancer and hedgehog in human holoprosencephaly, underscored how far reaching the results of these screens were.

Among the 126 genes that were identified was rhomboid, although the first rhomboid mutation was isolated some years earlier and was called veinlet, because it was a gene required for wing vein development, which is one of its post-embryonic functions. The embryonic phenotype of rhomboid placed it in the spitz group of genes comprised of spitz, Star, pointed, single-minded, and sichel (several derive their names from a misshaped head skeleton) (Mayer and Nusslein-Volhard 1988). The cloning revolution of the late 1980s resulted in the molecular identification of the spitz group genes: three membrane proteins and two transcription factors (Thomas et al. 1988; Bier et al. 1990; Rutledge et al. 1992; Scholz et al. 1993; Kolodkin et al. 1994). The only protein to have a domain signature that might suggest a molecular function was Spitz, which contained an epidermal growth factor (EGF) domain in its predicted extracellular region (Rutledge et al. 1992). Were the Spitz group proteins components of growth factor signaling?

\section{The importance of signal emission in development}

Cells learn their fates progressively during development, being instructed by local activating or inhibitory signals, or long-range morphogen gradients (Gerhart 1999). Surprisingly only about half a dozen conserved signaling pathways are responsible for orchestrating the hundreds of diverse cell communication events in development. Analysis of spitz group and EGF receptor (EGFR) mutant phenotypes implicated them in a single pathway that was involved in the development of virtually every tissue in the fly. The broad importance of EGFR signaling next focused attention on its regulation. Surprisingly, the EGFR and its ligand Spitz were found to be expressed in virtually every cell during development (Price et al. 1989; Schejter and Shilo 1989; Rutledge et al. 1992), but activation of the pathway was known to be highly dynamic in both space and time, as evidenced by staining for activated MAP kinase (Gabay et al. 1997), the main transducer of receptor tyrosine kinase signaling. How is signaling initiated at the required times and places?

Strikingly, only expression of the rhomboid gene precisely mirrors the activation of the pathway (Bier et al. 1990). Moreover, Rhomboid is the only limiting component: ectopic expression of only Rhomboid triggers EGFR signaling ectopically (Freeman et al. 1992; Sturte- vant et al. 1993). Removal of either Rhomboid or Star, another transmembrane Spitz group protein, recapitulates the null phenotype of Spitz, arguing that the EGF ligand Spitz is inactive in the absence of Rhomboid or Star (Mayer and Nusslein-Volhard 1988; Guichard et al. 1999). Finally, genetic mosaics established that Rhomboid and Star were required in the cell sending, not receiving, the signal (Golembo et al. 1996; zür Lage et al. 1997; Wasserman and Freeman 1998; Guichard et al. 1999; Wasserman et al. 2000), arguing that Rhomboid, with some involvement from Star, is the signal-generating component of EGFR signaling. But it remained unclear how Rhomboid and Star activate Spitz.

\section{The Spitz activation pathway}

Despite the success of Drosophila genetics in identifying the regulators of EGFR signaling, how signaling is activated at the molecular level could not be solved by this approach. Instead, biochemical analysis of Spitz in developing Drosophila embryos (Lee et al. 2001) and heterologous expression in a frog explant assay (Bang and Kintner 2000) revealed that Spitz is cleaved from its transmembrane anchor, and this required both the Rhomboid and Star proteins. Although these systems ultimately proved to be too complicated to reveal the molecular details of the signaling mechanism, Spitz processing could be recapitulated in transfected insect and mammalian cells (Lee et al. 2001; Pascall et al. 2002; Tsruya et al. 2002). Careful cell biological analyses by several groups revealed that Spitz is activated through regulated ligand trafficking and proteolytic cleavage (Fig. 1).

Signaling molecules like Rhomboid, Star, and Spitz were assumed to be at the cell surface, but surprisingly all three were found to reside in internal organelles (Lee et al. 2001; Pascall et al. 2002; Tsruya et al. 2002). Rhomboid is concentrated in the Golgi apparatus, while the EGF ligand Spitz is confined to the endoplasmic reticulum (ER). Star is distributed broadly, but its coexpression results in translocation of Spitz from the ER to the Golgi apparatus, where Spitz is cleaved from its transmembrane anchor, and secreted from the cell ready to initiate EGFR signaling in neighboring cells (Lee et al. 2001; Pascall et al. 2002; Tsruya et al. 2002). The sequence of events was revealed by pulse-chase experiments (Lee et al. 2001), and these observations in cultured cells were corroborated directly in Drosophila (Lee et al. 2001; Tsruya et al. 2002). Moreover, this model could be tested; a form of Spitz that could exit the ER by itself no longer relied on Star to allow cleavage and secretion from cells (Lee et al. 2001), and targeting Rhomboid to the ER allowed Spitz cleavage in the absence of Star (but cleaved Spitz was retained in the ER and not secreted) (Urban et al. 2002a).

These studies established that Star was the transport factor for Spitz, but it had no role in the cleavage event. So what is the Spitz protease, and what is the role of Rhomboid in this activation pathway? 


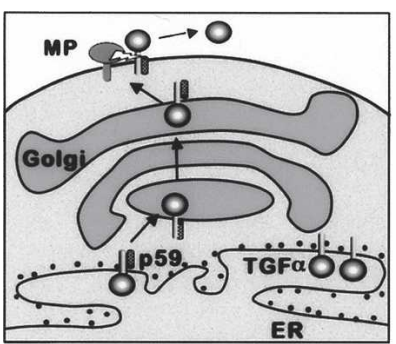

Vertebrates

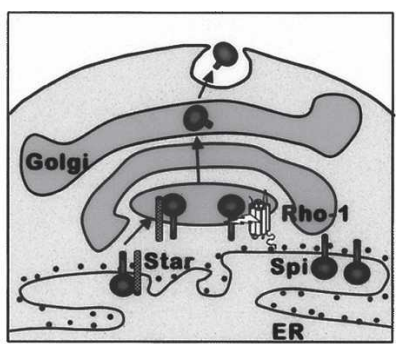

Drosophilia
Figure 1. Comparison of EGF ligand activation in vertebrates and Drosophila. (Left) In vertebrates, the EGF ligand TGF $\alpha$ is synthesized in the ER, and is transported through the secretory pathway by its association with cytosolic factors (including p59/GRASP55 and syntenin) that bind its C-terminal tail. Once it reaches the cell surface, TGF $\alpha$ is shed by ADAM-family metalloproteases (MP) by cleavage (depicted by a lightning bolt) in its juxtamembrane region, resulting in release of the active ligand domain. (Right) In Drosophila, the Spitz ligand is held in the ER until it complexes with the transmembrane protein Star. The Spitz-Star complex then migrates to the Golgi apparatus, where Spitz is cleaved (depicted by a lightning bolt) in its transmembrane domain by the intramembrane protease Rhomboid1. The cleaved ligand is then hyperglycosylated, palmitoylated, and secreted from the Golgi apparatus as an active ligand. Strikingly, although TGF $\alpha$ and Spitz are homologous proteins, the transport factors and proteases involved in their respective activation are unrelated.

\section{Putting a 'face to the name': Rhomboid is a protease}

Although Rhomboid does not resemble any known protease by global sequence analysis (Bier et al. 1990), the fact that Spitz cleavage occurs with similar efficiency in cell lines from very different organisms and tissues suggested that Rhomboid itself may be the Spitz protease (Urban et al. 2001). The obvious direct test of this model would be to determine whether pure Rhomboid protein could catalyze Spitz cleavage in vitro, but the difficulty in reconstituting activity with integral membrane proteins precluded this approach initially.

Instead, the cell-based Spitz cleavage assay was used to test a reformulated question: What type of protease might Rhomboid be (Urban et al. 2001)? Proteases are abundant, well-studied enzymes present in all kingdoms of life, with as much as $5 \%$ of the human genome predicted to encode proteolytic components (Lopez-Otin and Overall 2002). Many forms of these enzymes evolved independently, but resemble each other in their chemistry by virtue of convergent evolution. Brian Hartley (Hartley 1960) recognized this chemical similarity, and in 1960 proposed a grouping of all known proteases into what was then four catalytic classes: serine, cysteine, aspartyl, and metalloproteases (that use conserved histidines and glutamate to coordinate zinc ions). Although Rhomboid would be a novel protease, the basic chemistry of the hydrolysis reaction should conform to the known mechanisms.

Mutation of conserved residues in Rhomboid revealed that only six reduced strongly or abolished Spitz cleavage (Urban et al. 2001), four of which clustered in three dif- ferent transmembrane domains and resembled a serine protease-like catalytic triad and an oxyanion stabilizing site. Unexpectedly, these observations suggested that the Rhomboid catalytic apparatus was buried within the membrane bilayer, making Rhomboid the first described intramembrane serine protease (Fig. 2). In support of this model, Spitz was found to be the first growth factor cleaved in its transmembrane anchor, and this cleavage was sensitive only to a subset of serine protease inhibitors (Urban et al. 2001).

A second fundamental question was how specificity is

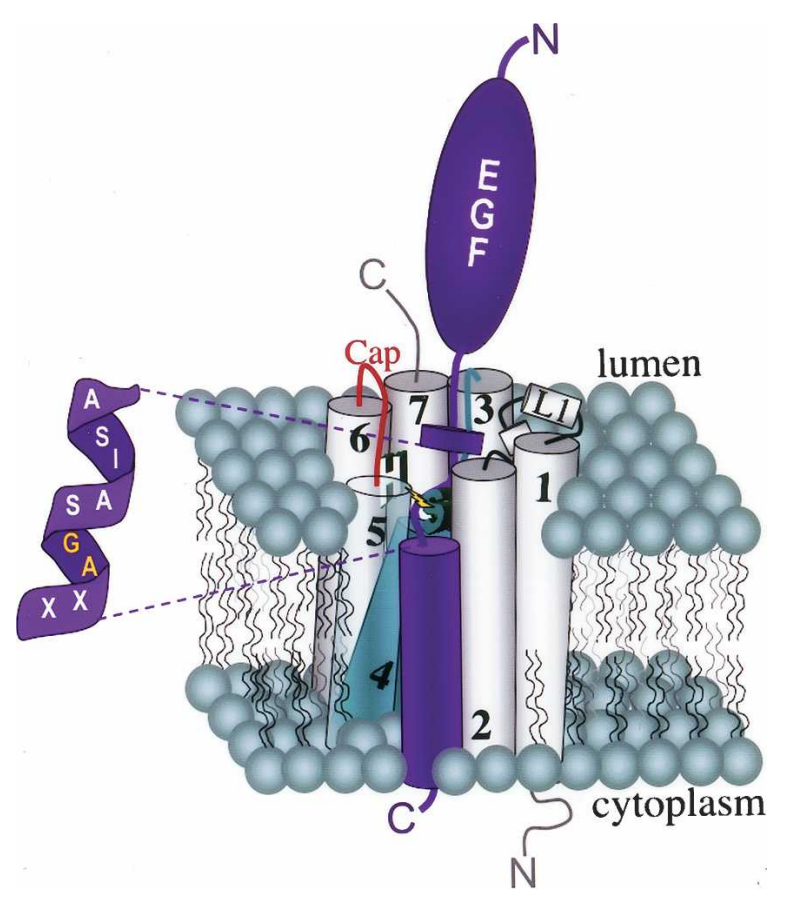

Figure 2. Molecular function of rhomboid. Drosophila rhomboid functions as a protease to activate EGFR signaling by catalyzing the intramembrane cleavage of the Spitz ligand. The model is based on the structure of a bacterial rhomboid, but note that the placement of the seventh transmembrane segment is arbitrary (since the bacterial rhomboid contained only six transmembrane domains). The rhomboid active site is embedded within the membrane, in a cavity formed by a central, recessed helix 4 (in cyan) and protected from lipid by a ring-like arrangement of helices (helix 5 has been rendered transparent to facilitate visualizing the interior). The loop between transmembrane segment 1 and 3 (L1) is submerged in the outer leaflet of the membrane to plug a gap between transmembrane segments 1 and 3. The active serine (cyan S) sits on top of the recessed helix 4 within a hydrophilic cavity, and is hydrogen bonded to the histidine (white $\mathrm{H}$ ) in transmembrane segment 6 . The L5 loop (in red), shown in the open conformation, is thought to cap the hydrophilic cavity and thus regulate water access to the active site. Substrate specificity relies on the sequence at the very top of the transmembrane domain, with residues known to confer helical flexibility being both necessary and sufficient for cleavage. In Spitz, this substrate motif is ASIASGA, with the GA (in yellow) being most important for cleavage. The substrate (in purple) is drawn on the outside of the protease ring, with the substrate motif being unwound into the active site in the interior of the enzyme, but this model is speculative. 
achieved: if Rhomboid is the Spitz protease, it should only cleave Spitz and not the hundreds of other transmembrane domains that it encounters in the cell. A mutational analysis mapped the critical region for Spitz proteolysis to the top of its transmembrane domain, in a region comprised of seven residues (Urban and Freeman 2003). This "substrate motif" was not only necessary, but sufficient for cleavage; placing just these seven residues into the transmembrane domain of other proteins converted them into Rhomboid substrates. A mutational analysis revealed that helix-relaxing residues are the key feature of this region, and the basis of Rhomboid specificity (Fig. 2). Since most transmembrane domains in the cell adopt model helices, Rhomboid substrates would be the small number of proteins that have more flexible helices. In this way, although substrates would certainly start out being helical in the bilayer environment, perhaps upon encountering Rhomboid the ease with which the helix could be unwound to facilitate cleavage might be the basis of specificity.

\section{Rhomboid proteins share conserved biochemical characteristics despite sequence divergence}

While it was expected that rhomboid proteins would be conserved in other animals, expanding sequence data revealed that rhomboid proteins are conserved in all kingdoms (with losses in a few lineages), being perhaps the most widely conserved membrane protein family known (Urban et al. 2001; Koonin et al. 2003). Sequence analysis also revealed a high degree of divergence at the protein level: the shared core region in rhomboid proteins consists of six transmembrane domains, but as a family they share only $\sim 5 \%$ sequence identity in this region (Urban et al. 2002b; Koonin et al. 2003). Further diversity results from highly variable $\mathrm{N}$ termini present in many rhomboid proteins, while the eukaryotic members have an additional transmembrane helix, added either N- or Cterminally to the core six.

Initially this suggested that rhomboid proteins have a more basic cellular function in bacteria, such as acting as transporters, with a signaling function in Drosophila being a more recent specialization (Rather et al. 1999; Wasserman et al. 2000; Mesak et al. 2004). Contrary to expectation, a first activity analysis indicated that rhomboid proteins from a variety of bacteria have similar enzymatic characteristics; most (but not all) rhomboid proteins from bacteria, invertebrates, and vertebrates tested could cleave Spitz, and required the Spitz substrate motif to catalyze this cleavage (Urban and Freeman 2003; Urban and Wolfe 2005). These initial analyses suggest that many rhomboid proteins share fundamental biochemical characteristics despite billions of years of evolution and extensive sequence divergence.

\section{Biochemical proof: molecular architecture} of an intramembrane serine protease

The important biochemical proof for this model was missing because no in vitro activity could be demonstrated with Rhomboid protein initially. Having learned more about the properties of these enzymes, it has recently been possible to express rhomboid proteins in bacteria (Lemberg et al. 2005; Maegawa et al. 2005; Urban and Wolfe 2005), purify them to apparent homogeneity, and analyze their activity against a pure recombinant substrate harboring the substrate motif from the Spitz transmembrane domain. While robust activity could be detected with several diverse rhomboid proteins, the substrate could not be cleaved by any rhomboid enzyme if the Spitz substrate motif was missing (Urban and Wolfe 2005), although $\gamma$-secretase (an unrelated intramembrane protease implicated in Alzheimer's disease) could readily cleave the proteins that do not contain the substrate motif. This analysis finally provided the missing evidence that rhomboid proteins directly catalyze intramembrane proteolysis, and further showed that they do so without the need for other auxiliary factors for either activity or specificity, in contrast to $\gamma$-secretase, which relies on other components for activity (Edbauer et al. 2003; Kimberly et al. 2003). This defined in vitro enzyme system provided the final proof that rhomboid proteins are novel proteases, and led to their inclusion into the EC database as the only family of intramembrane serine proteases (family EC 3.4.21.105). However, as with other enzymes, mechanistic insights into the function of rhomboid proteases would require structural analysis.

The crystal structure of one rhomboid ortholog has recently been determined, and provides the first highresolution glimpse of any intramembrane protease (Wang et al. 2006). The resulting structure corroborates many prior functional analyses and proposed mechanisms, while revealing important new features. As suspected, the general architecture of the protein is a ring of transmembrane helices, but surprisingly, the long L1 loop between transmembrane segments 1 and 2 dips into the outer leaflet of the bilayer to close a V-shaped gap between helices 1 and 3 (Fig. 2). Interestingly, the catalytic serine is at the top of the transmembrane segment 4 , but this helix does not traverse the bilayer completely, instead being sunk $\sim 10 \AA$ from the extracellular face. This creates a large central hydrophilic cavity that opens to the extracellular side, with its bottom apex being the catalytic serine and conserved transmembrane residues lining the hydrophilic pocket. The cavity in the structure contains several water molecules that would be required for catalysis, but access appears to be regulated by a mobile extracellular loop (termed the Cap). The active site serine and histidine residues interact via a strong hydrogen bond in the interior of the protease ring, and the planar ring of the histidine is aligned by stacking onto the tyrosine, thus obviating the need for a third catalytic triad residue, as is required in classical serine proteases. Indeed, the transmembrane asparagine-first proposed to be the third member of the catalytic triadis on the other side of the serine. Intramembrane proteolysis is therefore accomplished by a catalytic serinehistidine dyad in a hydrophilic environment within the membrane bilayer that is protected from lipids by surrounding protein segments. This analysis also indicated 
that, while trimers were present in the crystal, rhomboid proteins probably function as monomers, with each molecule functioning independently and not as obligate multimers.

The substrate is predicted to access the internal rhomboid active site laterally, but the precise point of entry is currently not known. The cavity between transmembrane segments 1 and 3 is plugged by the L1 loop, and it has been proposed that movement of this loop could open access to the active serine (Wang et al. 2006). However, the serine points away from this possible site of entry, thus the substrate or the serine would need to turn for cleavage to occur. Alternatively, other points of entry are also conceivable, and further analysis is required to differentiate between the various possibilities. In any case, the substrate enters the active site laterally through a narrow opening on rhomboid, presumably by local melting and extension of the top of the substrate transmembrane segment into the internal active site. This idea is nicely supported by prior mutagenesis experiments that implicated the top third of the substrate transmembrane domain to be sufficient for cleavage, and found that this region is comprised of helix-relaxing residues (Urban and Freeman 2003). Importantly, although the precise site of substrate entry and sequence of events in intramembrane proteolysis remain incompletely understood, the current structures provide a framework for further experimental scrutiny, both at the functional and structural levels.

\section{Intramembrane proteolysis is a widespread regulatory mechanism}

Rhomboid enzymes are not alone in catalyzing intramembrane proteolysis: three other examples of intramembrane proteolysis have been described as a newly emerging signaling paradigm (Brown et al. 2000; Steiner and Haass 2001; Urban and Freeman 2002; Weihofen and Martoglio 2003; Wolfe and Kopan 2004). Site-2 protease was the first such intramembrane protease to be identified, and functions as a metalloprotease in regulating cholesterol and fatty acid biosynthesis (Rawson et al. 1997). Presenilin was discovered to be the catalytic component of $\gamma$-secretase, an enzyme that plays a central role in Alzheimer's disease by providing the final cleavage that generates the Amyloid- $\beta$ peptide (Wolfe et al. 1999). A more recent addition is signal peptide peptidease, a presenilin-type protease that cleaves signal peptides after they have been removed from precursor proteins (Weihofen et al. 2002). One fundamental difference between rhomboid and other intramembrane proteases is the function of the domains released from the membrane; rhomboid enzymes usually release factors to the outside of the cell, while other intramembrane proteases typically release factors into the cytosol (often transcription factors) as the ultimate signal effectors in the cells receiving the signal (Urban and Freeman 2002).

Animals are known to use different signaling pathways compared with plants and prokaryotes; therefore, the wide conservation of rhomboid proteins raises the exciting possibility that they might be a common link between diverse communication pathways. Recent analyses now indicate that, while their biochemical properties remain similar, rhomboid enzymes also function in many different processes. The roles can be divided into two broad categories: a prominent function in signaling between cells during development of various organisms, and roles within a cell that have implications for human health.

\section{Rhomboid proteins in development}

Conservation of function within an organism: a family affair in flies

The best understood biological function for any rhomboid protease is in EGFR signaling during Drosophila development, but the situation is more complex than initially suspected. Sequencing of the Drosophila genome revealed that many developmental genes exist in families, with Rhomboid being part of a family of seven rhomboid-like genes in Drosophila, while rhomboid gene families also exist in other multicellular organisms (Guichard et al. 2000; Wasserman et al. 2000). At the time the genome was sequenced, only the original rhomboid gene (renamed rhomboid-1) had been understood on any meaningful level. Why does Drosophila contain seven rhomboid genes, and what do the other homologs do?

While it was possible that other rhomboid proteins could be activators of different signaling pathways, currently the characterized members function as tissue-specific activators of EGFR signaling. The two rhomboid genes most similar to rhomboid-1-rhomboid-2 and rhomboid-3-are arranged as a gene cluster on chromosome 3, probably reflecting evolution by gene duplication and divergence. All three have very similar biochemical properties, but are expressed in different tissues, suggesting that the task of transcribing a single rhomboid gene in the $>60$ different EGFR signaling contexts was split among multiple genes whose protein products carry out similar biochemical roles (Urban et al. 2002a). In fact, transcriptional control is currently the only known source of rhomboid regulation in Drosophila. Genetic analyses have shown that rhomboid genes have distinct but partly overlapping functions as tissue-specific EGFR regulators.

Rhomboid-3 is most similar to Rhomboid-1, and its main role is in eye development. Although EGFR signaling induces the fate of all cell types in the developing eye, the genetic removal of rhomboid-1 during eye development resulted in no defects (Freeman et al. 1992). This mystery was ultimately resolved when a genetic analysis revealed that rhomboid-3 fulfills the major role for a rhomboid during eye development (Wasserman et al. 2000). In fact, both rhomboid-1 and rhomboid-3 are expressed in the developing eye and probably function together (although rhomboid-3 has the predominant role), since a double mutant has a more severe phenotype than either alone. rhomboid-3 was actually found to be the gene mutated in the classical fly mutant roughoid that was isolated in the early 20 th century. Rhomboid-3 
also has roles during embryogenesis, including in tracheal tube migration (Gallio et al. 2004) and in protecting epidermal cells from apoptosis (Urban et al. 2004), but both are through regulating EGFR signaling.

Rhomboid-2 is expressed in the germline, where it acts to promote differentiation of germline stem cell progeny (Guichard et al. 2000; Schulz et al. 2002). Germline stem cells must be encapsulated by somatic cells to differentiate into gametes, and this encapsulation process requires signaling from the germ cells to the neighboring somatic cells. A genetic analysis revealed that rhomboid-2 (also called stet, for stem cell tumor) acts in the germ cells, presumably to activate a signal that is sent to the somatic cells. In rhomboid-2 mutants, this signal is thus not sent, somatic cells do not encapsulate the germ cells, and the germ cells fail to differentiate and accumulate as germline stem cells and their early progeny. Targeted expression of either Rhomboid-2, or notably Rhomboid-1 in germline stem cells rescued this phenotype, further indicating that these two factors have highly similar biochemical characteristics. However, the ligand responsible remains unknown, since activated forms of both the Spitz and Gurken EGF ligands failed even to modify the rhomboid-2 defect. Moreover, it is not known whether Rhomboid-2 has a function late in oogenesis, when Gurken is required to signal to the overlying somatic follicle cells (Roth et al. 1995).

Drosophila contains four other rhomboid-like genes (Guichard et al. 2000; Wasserman et al. 2000), and while a recent genetic analysis has revealed that Rhomboid-7 functions in mitochondrial dynamics (as discussed later) (McQuibban et al. 2006), the precise roles of the other three remain unknown. Rhomboid-4 and Rhomboid-6 resemble Rhomboid-1, Rhomboid-2, and Rhomboid-3, and at least Rhomboid-4 can cleave all transmembrane EGF ligands in a cell-based assay, and can activate EGFR signaling in developing tissues (Urban et al. 2002a). Rhomboid-5 does not contain the active site serine, and is thus unlikely to function as an intramembrane protease, but could have a regulatory role. Further analysis is required to determine the function of these rhomboidlike genes.

\section{Rhomboid proteins in other invertebrates: playing a supporting role in worms}

While rhomboid proteins act as key activators of EGFR signaling in Drosophila, current evidence suggests that they play surprisingly minor roles in EGFR signaling in other animals, even in other invertebrates. Caenorhabditis elegans contains five rhomboid-like genes, with ROM-1 bearing the strongest resemblance to Drosophila Rhomboid. Surprisingly, a genetic deletion mutant of ROM-1 resulted in no phenotype at all, whereas the null mutant of the EGF ligand LIN3 is embryonic lethal, indicating that ROM-1 is not essential for EGFR signaling (Dutt et al. 2004).

Instead, it was discovered through a series of elegant genetic interaction experiments that ROM-1 has a minor role in amplifying EGFR signaling during vulval devel- opment (Fig. 3). The initial EGF signal comes from the AC cell, and induces the underlying Pn.p cells to adopt one of three fates. Contrary to expectation, ROM-1 is not expressed in the AC cell, the source of the signal, but is actually a gene activated by high EGFR signaling in Pn.p precursors. However, the cells expressing ROM-1 become a new source of the LIN3 ligand, and are thought to contribute positive feedback amplification of the original AC LIN3 signaling event. Intriguingly, the LIN3 gene encodes two splice variants, with only the longer variant being dependent on ROM-1 for signaling. The longer variant introduces 15 residues just upstream of the transmembrane domain, but since these were genetic interaction experiments, it was not possible to determine whether the 15-residue insertion directly affects the ability of the LIN3 ligand to serve as a ROM-1 substrate, or affects it function by indirect means (such as altering its subcellular localization).

A necessary caveat of these experiments is that only two rhomboid-like genes, ROM-1 and ROM-2, were analyzed; the remaining three worm ROMs, although more distantly related, contain the elements necessary for activity and therefore some level of redundancy might be expected. Moreover, the full functions of rhomboid-like genes in worms remain unexplored; ROM-1 is expressed widely, but a phenotype in vulval development was noticed only in a sensitized vulva mutant background. It remains possible that other phenotypes would become evident in other sensitized backgrounds; for example, during embryogenesis. But the subtle role of rhomboid proteins in regulating EGFR signaling in the vulva, one of the most prominently studied EGFR signaling contexts, is surprising compared with its central role in Drosophila.

\section{The enigma of rhomboid proteins in vertebrates}

Despite considerable interest and effort, the role of rhomboid-like genes in vertebrates remains unknown.

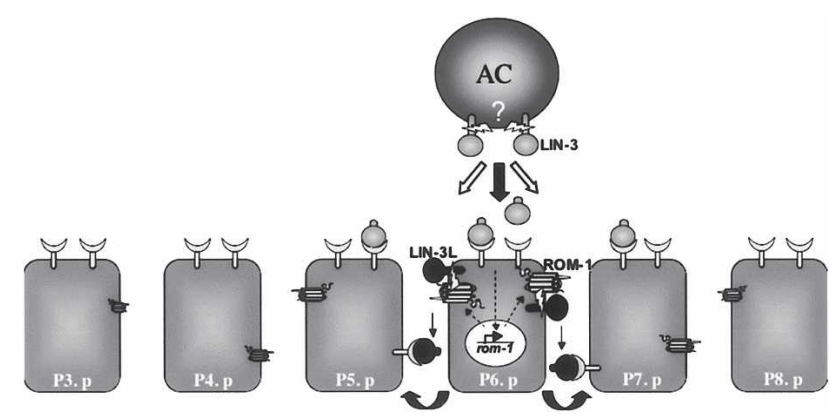

Figure 3. ROM-1 functions to amplify EGF signaling during $C$. elegans vulva development. LIN-3 is the transmembrane EGF signal that is sent from the anchor cell $(\mathrm{AC})$ to the underlying Pn.p cells to induce vulval fates. ROM-1 has no function in this initial signaling event, which is probably mediated by an unknown protease (question mark in AC cell), but high levels of signaling induce expression of ROM-1 in the signal-receiving Pn.p cells. The ROM-1 protein is then thought to catalyze cleavage of the longer isoform of LIN-3 (in black), providing a new source of signal as a positive feedback relay of EGF signaling. 
Vertebrates contain at least six clear rhomboidlike genes (named RHBDLs for rhomboid-like), but superficially they can be grouped in three major classes. RHBDL1, RHBDL2, and RHBDL4 (also termed RHBDL3 in some annotations) contain the residues necessary for catalysis, while RHBDL5 and RHBDL6 are missing the active site serine and are thus likely to be catalytically inactive. The final class is PARL, a single rhomboid protein that localizes to mitochondria where it functions in membrane dynamics (discussed later). There could be at least four other rhomboid-like proteins in humans, but these have unusual topologies and their homology with rhomboid is limited to smaller regions.

Initially an involvement of vertebrate rhomboid enzymes in regulating EGFR signaling seemed probable by analogy, but none of the presumably active RHBDL proteins can cleave any of the seven human EGF ligands tested in a cell-based assay (Lohi et al. 2004; Pascall and Brown 2004; Nakagawa et al. 2005). It should be noted that a few known EGF ligands escaped this analysis, and that it remains possible that unidentified factors were missing in these assays that could be important for the trafficking and/or cleavage of these EGFR ligands. These caveats notwithstanding, it is unlikely that active RHBDL proteases have a major role in regulating EGFR signaling in vertebrates; compelling genetic and biochemical evidence indicates that this role is fulfilled by ADAM family metalloproteases (see Fig. 1; Peschon et al. 1998; Sahin et al. 2004). A more specialized role for RHBDLs, like that observed in worms, remains possible. So what might be the major role of rhomboid enzymes in vertebrates?

The only vertebrate rhomboid whose proteolytic activity has been demonstrated directly is RHBDL2, the human, mouse, and zebrafish orthologs of which have the same substrate specificity for Spitz-like molecules as the Drosophila enzymes (Urban and Freeman 2003). This unexpected observation facilitated a search for proteins that resemble the Spitz substrate motif in their transmembrane domains, and might thus be physiological RHBDL2 substrates. Two groups used this approach, and subsequently found two different possible targets.

Pascall and Brown (2004) found that B-type ephrins are very efficient substrates for RHBDL2. Ephrin receptors are the most abundant receptor tyrosine kinases in vertebrates, and have been implicated in various aspects of brain development, including restricting neuronal migration (Wilkinson 2001). Interestingly, B-type ephrins are thought to require membrane attachment to signal, implying that RHBDL2 cleavage might terminate signaling, perhaps to allow the migrating cell to turn away from the repulsive signal. A second protein identified in these searches was thrombomodulin, a type I glycoprotein whose precise function is unknown but has been implicated in blood clotting (Lohi et al. 2004). While a circulating form of thrombomodulin is known to exist, it is not clear if it is released by proteolysis, and whether this form has physiological significance. A possible role for RHBDL2 in nervous system development via ephrin signaling, or blood clotting via thrombomodulin, remains unknown.
A developmental function for at least one vertebrate rhomboid is suggested by its dynamic expression pattern during mouse embryogenesis. This gene, termed RHBDL4 or Ventrhoid, is expressed primarily in ventral neuronal tissues, with the highest expression being in the spinal nerve cord (Jaszai and Brand 2002). However, neither a knockout phenotype nor putative substrates have been identified to reveal a potential developmental function for Ventrhoid.

Many protease families contain recognizable members that do not have proteolytic activity, and progress has recently been made in characterizing the noncatalytic class of rhomboid proteins. RHBDL5 was found to associate physically with the EGF ligands TGF $\alpha$ and HB-EGF in human cells, and could activate EGFR signaling when misexpressed in developing Drosophila wings (Nakagawa et al. 2005), like other Drosophila rhomboid proteins. Whether this was indeed direct activation of the EGFR pathway or other pathways remains unclear, but it does raise the possibility that noncatalytic rhomboids have functions in regulating signaling beyond acting as intramembrane proteases, perhaps by modulating the activity of catalytic rhomboid proteins. A prediction of these observations is that a noncatalytic RHBDL mouse knockout would have TGF $\alpha$-like epidermal hair patterning defects, although this knockout is not yet available (Luetteke et al. 1993; Mann et al. 1993).

\section{Rhomboid proteins in other kingdoms: ancient cell communication pathways?}

rhomboid-like genes are also conserved in all of the other kingdoms of life, including in plants, fungi, bacteria, and archaea (Wasserman et al. 2000; Koonin et al. 2003), and although their functions remain largely unknown, early observations also suggest at least some involvement in cell signaling. Plants are not thought to have evolved EGFR-type signaling (although receptor kinases have been identified), but Arabidopsis thaliana contains over a dozen rhomboid-like genes. An activity analysis revealed that at least one Arabidopsis rhomboid was active against Spitz and was localized to the Golgi apparatus in plant cells (Kanaoka et al. 2005), suggesting that some of the features of rhomboid-mediated intramembrane proteolysis are present in plants. Several rhomboid-like genes appear to be ubiquitously expressed during development and available mutant plants have not yet displayed recognizable phenotypes, suggesting that redundancy between different rhomboid genes could complicate a genetic analysis aimed at studying this gene family.

A particularly enigmatic early observation was the presence of rhomboid genes in almost all sequenced prokaryotic genomes. It was presumed that their function represented an ancestral, nonsignaling role such as membrane transport (Mesak et al. 2004). But an early analysis in Providencia stuartii, a Gram-negative human pathogen of the urinary tract, indicated the opposite. Studies aimed at understanding antibiotic resistance led to the discovery that Providencia monitors its population size 
through intercellular communication, like many bacterial species (Rather et al. 1997; Waters and Bassler 2005). As the population grows, signals are secreted and accumulate, regulating a subset of genes once a threshold has been reached. This process, termed quorum sensing, allows different genes to be expressed at low versus high population densities by querying the cell population. Two separate genetic screens for factors regulating the expression of quorum sensing target genes identified a gene, called aar $A$, that was required for production or transport of an unknown Providencia signal in the sending cell (Fig. 4; Rather and Orosz 1994; Rather et al. 1999). It was noticed some years later that AarA is homologous to Drosophila rhomboid (Gallio and Kylsten 2000).

This intriguing observation was confounded by the fact that Gram-negative bacteria like Providencia typically use a variety of autoinducers for quorum sensing, especially $\mathrm{N}$-acyl modified homoserine lactones and furanosyl borate diesters, but at the time were not thought to use proteins or peptides (Waters and Bassler 2005). Peptides were thought to be limited to quorum sensing by Gram-positive bacteria. Thus, in the absence of knowing the identity of the Providencia signal or the molecular function of AarA in signal production, the possible significance of this signaling system to that in Drosophila remained unclear.

Strikingly, it was shown that AarA could partially rescue defects associated with both rhomboid-1 and rhomboid-3 mutants during wing and eye development, respectively, in Drosophila, while the Drosophila protein could partly restore the production of the quorum-sensing signal in aarA-mutant Providencia (Gallio et al. 2002). Consistent with these findings, a mechanistic analysis revealed that AarA functions as a protease and relies on the "substrate motif" residues in Spitz for cleavage (Urban et al. 2002b; Urban and Freeman 2003; Urban and Wolfe 2005). These unexpected observations suggested that this signal-generating mechanism might be the same as in Drosophila, and the first known to be conserved between bacteria and animals.

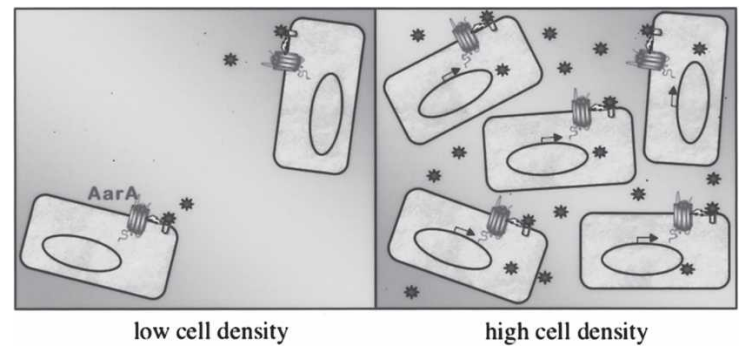

Figure 4. Rhomboid function in bacterial quorum sensing. Many bacterial species monitor their population density by releasing signals that, when they reach a threshold concentration, regulate the expression of genes appropriate for higher population size (shown at right). The pathogen Providencia uses the rhomboid-like protein AarA for sending the unidentified signal (designated by a asterisk) for quorum sensing. This signal is known to represses certain genes, while activating others.
However, whether Providencia represents a new paradigm in bacterial signaling, or a rare exception remains unknown. The AarA-dependent signal in Providencia still has not been identified. Although genetic knockouts of bacterial rhomboid-like genes have recently been made in Escherichia coli and Bacillus subtilis, resulting in no obvious phenotype (Maegawa et al. 2005) and a filamentous phenotype (Mesak et al. 2004), respectively, they have not yet revealed a precise molecular function for these bacterial rhomboid enzymes. Given the diversity of microbial life and the precedent of rhomboid enzymes regulating several unrelated processes in animals, it seems possible that they will also have functions beyond quorum sensing in bacteria.

\section{Rhomboid proteins in human disease}

\section{The mitochondrial connection}

A phylogenetic analysis indicates that eukaryotic rhomboid-genes fall into two major categories: the large RHO family and the smaller PARL family, which typically is represented by one member in each eukaryotic species (Koonin et al. 2003). Recent progress has clarified the role of PARL, as well as implicated this rhomboid protease in human disease.

PARL, presenilin-associated rhomboid-like, derives its name from being identified in a yeast two-hybrid screen for proteins that could interact with Alzheimer's presenilin protein (Pellegrini et al. 2001), although this association does not occur physiologically. The first functions of a PARL protease were discovered in the budding yeast: early genome-wide screens revealed that its homolog of PARL, Pcp1 (also called Rbd1 and Ygr101w), is localized to mitochondria (Steinmetz et al. 2002), and its deletion results in mitochondrial fragmentation (Dimmer et al. 2002), but the basis was unclear. Pcp1 was subsequently discovered to be responsible for cleaving the targeting sequence of cytochrome $\mathrm{c}$ peroxidase (Ccp1), a nuclear-encoded mitochondrial protein present in the intermembrane space (Esser et al. 2002). This defined a fourth and previously unknown targeting peptide processing pathway in mitochondria. However, pcp1-deleted cells had a slow growth phenotype while the ccp1 deletion did not, suggesting that the basis of the $p c p 1$ phenotype results from failure to process another substrate.

In a search for Pcp1 substrates, deletions of single-pass membrane protein candidates were tested for a pcp1like slow growth phenotype (McQuibban et al. 2003). This identified Mgm1, a transmembrane dynamic-like GTPase, as a possible substrate, and revealed mitochondrial fragmentation as the basis for the slow growth defect of both mgm1- and pcp1-deleted cells. Mitochondria in many organisms exist as a tubular network that results from regulated mitochondrial fusion and fission events, and Mgm1 is essential for fusion of both mitochondrial membranes (Sesaki et al. 2003b; Wong et al. 2003). Mgm1 exists in two isoforms, a long form that crosses the inner membrane, and a short form that is 


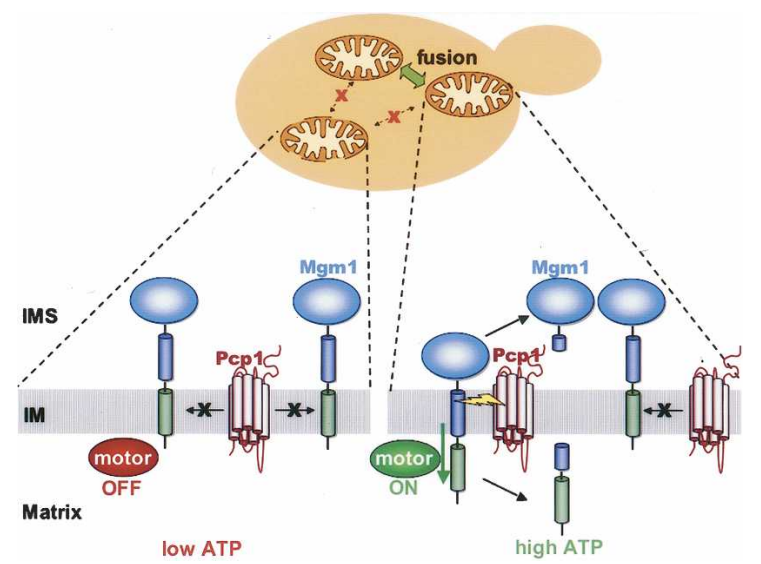

Figure 5. Model of rhomboid function in regulating mitochondrial membrane dynamics. Mitochondrial membrane fusion requires both the full-length and cleaved form of the Mgm1 GTPase. (Left) Under lower ATP concentrations, the first hydrophobic segment is preferentially used as the Mgml transmembrane anchor, which cannot be cleaved by Pcp1. In this way, unhealthy mitochondria (lower mitochondrion) are prevented from fusing with healthy mitochondria. Stationary phase conditions also result in no Pcp1 expression, and thus no Mgm1 cleavage. (Right) Under conditions of Pcp1 expression and high ATP levels, the second hydrophobic segment is used as the Mgm 1 transmembrane domain to a higher degree, facilitating cleavage by Pcp1. This cleaved form of Mgm1 acts in concert with the uncleaved form to promote mitochondrial membrane fusion (upper mitochondria). It should be noted that the exact topology of Pcp1 in the inner membrane, including whether Pcp1 has six or seven transmembrane domains, has not been resolved.

released by Pcp1 proteolysis into the intermembrane space. Failure of Mgm 1 to be processed in a pcp1 deletion verified it to be the Pcpl substrate most likely to be responsible for the $p c p 1$ deletion phenotype. Reciprocally, Pcp1 was identified in a specific search for the Mgm1 processing protease (Herlan et al. 2003), and was also isolated independently in a screen as ugo2, a gene required for mitochondrial membrane fusion (Sesaki et al. 2003a).

Both cleaved and uncleaved forms of Mgm1 are required for membrane fusion, and one intriguing model suggests that the ratio is dictated by ATP levels in the mitochondrial matrix through a process termed alternative topogenesis (Fig. 5; Herlan et al. 2004). Mgm1 has an $\mathrm{N}$-terminal targeting sequence that mediates its import into mitochondria, followed by two hydrophobic sequences. The first acts as a transfer stop signal and generates the long form of Mgm1, and this transmembrane sequence is thought not to be a substrate for Pcp1. Under high ATP conditions, an ATP-dependent import motor pulls Mgm1 further in, resulting in a stop at a second hydrophobic sequence, which is a substrate for Pcp1. Cleavage of this second hydrophobic stretch generates the short form. Herlan et al. (2004) hypothesize that in low ATP levels, as might occur in damaged mitochondria, the short form is not generated, excluding damaged mitochondria from fusing with healthy mitochondria.
Interestingly, the human homolog of $m g m 1$ is OPA1, a gene mutated in autosomal-dominant optical atrophy, the most common early onset form of blindness (Alexander et al. 2000; Delettre et al. 2000). OPA1 is widely expressed in the body, but highest levels are present in the retina, a high-energy requiring tissue. Haplo insufficiency is thought to cause mitochondrial dysfunction, leading to retinal ganglion cell and optic nerve degeneration, resulting in blindness within the first two decades of life. Consistent with this model, reducing OPA1 expression using siRNAs results in fragmented mitochondria as well as increased cell apoptosis (Olichon et al. 2003; Griparic et al. 2004; Lee et al. 2004). A detailed examination subsequently revealed that in addition to its role in mitochondrial membrane fusion, OPAl also has a completely independent function as an antiapoptotic factor (Frezza et al. 2006). In this role, OPAl is thought to oligomerize in order to hold cristae together at different membrane anchor points (Fig. 6). Disruption of these oligomers, as occurs during apoptosis, or by ablating OPA1, releases cytochrome c from cristae and activates apoptosis independent of mitochondrial fragmentation.

Surprisingly, a $P A R L$ knockout mouse revealed that PARL is not involved in mitochondrial fusion, but solely participates in OPA1's function as an antiapoptotic factor (Cipolat et al. 2006). The cleaved form of OPA1 is only required for its function in tethering cristae, not for mitochondrial membrane fusion (Cipolat et al. 2006; Frezza et al. 2006). In a PARL knockout mouse, mitochondria suffer no fragmentation, but the cristae are more open, and release cytochrome c more readily (Fig. 6). This sensitizes many different cell types to apoptosis triggered by intrinsic, but not extrinsic, cues. As a result,

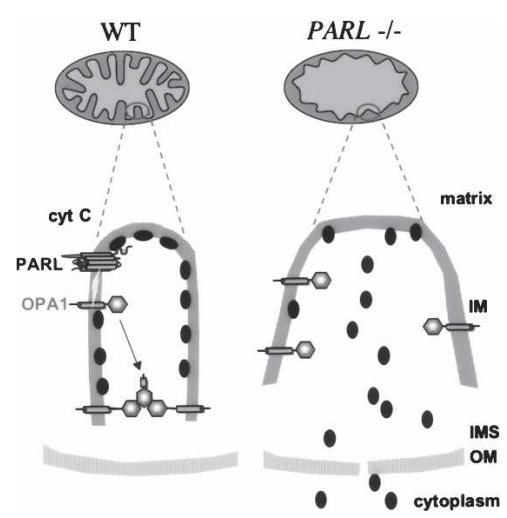

Figure 6. Role of PARL in maintaining tight mitochondrial cristae in vertebrates. PARL cleaves OPAl (gray shape with hexagonal end), which associates with transmembrane OPA1 molecules to form oligomers that maintain tight mitochondrial cristae and prevent cytochrome c (black ovals) release into the cytosol. In a PARL knockout mouse, OPA1 cleavage is reduced, which reduces OPA1 oligomers that maintain tight cristae. The mitochondrial cristae are more open, which facilitates cytochrome c release and sensitizes cells to intrinsic apoptotic stimuli. Note that PARL and OPA1 were also found to associate physically and may thus be in a complex (not shown in diagram), presumably prior to cleavage. 
the mice are born normally but at $\sim 4$ wk of age suffer severe growth retardation, and progress through multisystemic atrophy, resulting in gross muscle, thymus, and spleen loss, leading to death.

Although it is striking that the function of PARL is different in yeast and mice, being mitochondrial membrane fusion and antiapoptosis through cristae remodeling, respectively, the actual biochemical role of PARL is similar in both contexts, while the outcome appears different. In both cases, PARL cleaves a transmembrane GTPase to release it into the intermembrane space. In the case of Mgm1, the cleaved form is essential for mitochondrial membrane fusion, while in the case of OPA1, the cleaved form modulates cristae folding and thus protects from cytochrome c release. In support of this commonality, expressing human PARL in a pcp1deleted yeast partially rescued the mitochondrial fragmentation defect. In fact, in addition to mitochondrial fragmentation, there may be a cristae defect in $p c p 1 \mathrm{mu}-$ tant yeast (Amutha et al. 2004), but a prominent antiapoptotic role may be a more recent evolutionary development, since removing Drosophila PARL (Rhomboid-7) or OPAl-like with mutations or RNA interference (RNAi) resulted in fragmented mitochondria but no increase in apoptosis (McQuibban et al. 2006).

While OPA1 oligomerization is the regulation point by which $\mathrm{BH} 3$ proteins disrupt OPA1 association and thus open cristae to release cytochrome c, it is not clear whether OPAl cleavage by PARL is regulated. Importantly, regulation of OPA1 cleavage could also be exerted on a different protease, since OPAl cleavage is reduced by only twofold in a PARL knockout mouse, implying the existence of other OPA1-processing (or compensating) proteases. A biochemical study has also indicated that PARL may have a function beyond its proteolytic activity; the $\mathrm{N}$-terminal domain of PARL was found to be cleaved, and the released $\mathrm{P} \beta$ peptide entered nuclei in a potentially regulated manner (Sik et al. 2004). The implications of this observation are currently under investigation.

The possible linkage of PARL as a candidate disease gene has recently been explored, with a surprising outcome unrelated to optical atrophy. Mitochondrial dysfunction in skeletal muscle is thought to be an important factor in obesity and type II diabetes. A search for genes differentially expressed in skeletal muscle mitochondria of diabetic versus normal rats identified 54 genes, but only one was located in a chromosomal region linked to diabetes in humans (Walder et al. 2005). This gene was $P A R L$, and it showed decreased expression in obese and diabetic rats, but an exercise regimen that improved their pathology also resulted in an increase of $P A R L$ expression. Subsequent analyses in human subjects revealed a correlation between PARL expression and insulin sensitivity, and in a second analysis, one $P A R L$ variant and insulin levels, a surrogate marker of type II diabetes. Intriguingly, Pcp1 was also found to be down-regulated in yeast that have become metabolically less active as they entered stationary phase (McQuibban et al. 2003). It is presently unclear how PARL deficiency might result in diabetes, and whether this function involves OPA1. The newly generated PARL mouse knockout model should be valuable in deciphering the mechanism, and indeed in evaluating whether PARL is the disease susceptibility gene at 3q27 implicated in obesity and diabetes.

\section{Other vertebrate rhomboid proteins in human disease}

Past the precedent of PARL, little is known about the involvement of RHBDLs in human disease. If RHBDLs function in regulating EGFR signaling, it would be expected that they might be proto-oncogenes; increased EGFR signaling has been implicated in the formation of various epithelial tumors, including lung and breast cancer (Harari 2004). It also remains possible that other RHBDLs that are expressed during development will be implicated in congenital defects.

\section{Rhomboid enzymes in parasitic diseases: co-opting signaling mechanisms?}

An exciting new direction of research has been the implication of parasite-encoded rhomboid enzymes in devastating human diseases such as malaria and toxoplasmosis. Malaria affects $10 \%-40 \%$ of the world's population, causing $>1$ million deaths every year (Greenwood and Mutabingwa 2002), and has become the most common cause of death in children under the age of $5 \mathrm{yr}$ in several developing countries. Toxoplasmosis is caused by Toxoplasma gondii infection of immunocompromised individuals or during pregnancy, and is the most common cause of neurologic birth defects in several Western countries (Carruthers 2002).

These protozoan pathogens are obligate intracellular parasites that must invade host cells not only to establish an infection, but to survive. This exquisite sensitivity has made understanding the parasite invasion machinery an important therapeutic goal. Invasion of host cells is an active process mediated by the parasite's transmembrane adhesin molecules (Fig. 7), which are secreted from internal organelles called micronemes onto the apical surface (Carruthers et al. 1999; Huynh et al. 2003; Soldati et al. 2004). The adhesins then establish junctions with the host cell surface receptors on the outside, and link to the underlying parasite actin-based cytoskeleton on the inside of the parasite (Kappe et al. 1999; Jewett and Sibley 2003). Motors push these moving junctions to the posterior of the parasite, forcing entry of the parasite into an invaginating vacuole made from the host cell plasma membrane (Dvorak et al. 1975; Aikawa et al. 1978). Once the adhesin-receptor complexes reach the posterior of the parasite, they are released by proteolysis, allowing the host cell membrane to seal and resulting in the internalization of the parasite enclosed within the parasitophorous vacuole. Parasite replication and development ensues, protected within the parasitophorous vacuole, forming progeny that are ultimately released by destruction of the host cell by lysis (Glushakova et al. 2005). 
Figure 7. Parasite-encoded rhomboid enzyme functions in host cell invasion. Protozoan parasites of the phylum Apicomplexa store adhesins essential for host cell invasion in internal microneme organelles. During invasion, these adhesins are translocated onto the apical surface, and engage cellular receptors on the outside, while linking to the actomyosin cytoskeleton on the inside of the parasite (dashed line). The resulting junctions are motored toward the posterior end of the parasite, pushing the parasite into the invaginating vacuole. Colocalization of the adhesin complexes with Toxoplamsa ROM5 at the posterior of the parasite leads to cleavage of MIC adhesins in their transmembrane domains, resulting in junction dissolution and parasite internalization.

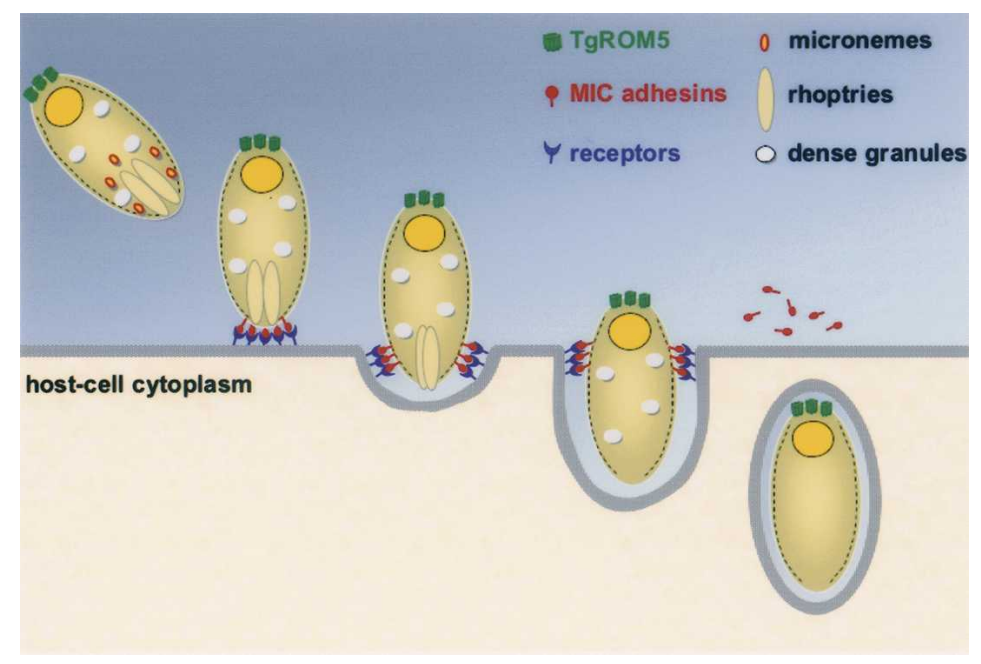

Although adhesin cleavage at the end of invasion is thought to be essential, the proteases responsible had not been identified. Among the best studied adhesins is MIC2 of Toxoplasma, and the protease responsible for its release from cells was termed MPP1, microneme protein protease 1 (Carruthers et al. 2000). The properties of MPP1 were unusual; it was not inhibited by most common protease inhibitors except dichloroisocoumarin (DCI) (Carruthers et al. 2000), and cleavage of one adhesin, MIC6, was initially mapped to its transmembrane segment (Opitz et al. 2002). A search for rhomboid substrates beyond Drosophila identified the Spitz substrate motif within MIC adhesins in Toxoplasma, including MIC2 and MIC6, and this observation led to the proposal that a parasite rhomboid protease might be the MPP1 activity during invasion (Urban and Freeman 2003). Subsequently, MIC2 was also found to be cleaved within its transmembrane anchor (Zhou et al. 2004).

A biochemical analysis revealed that one Toxoplasma rhomboid protease in particular most likely fulfills the role of MPP1 (Brossier et al. 2005). Toxoplasma encodes six rhomboid-like genes, one of which is homologous to PARL, and thus not involved in cell surface proteolysis (Dowse and Soldati 2005). Activity analysis of the remaining five revealed that four are active proteases that share substrate specificity with Drosophila rhomboid enzymes (Brossier et al. 2005). However, only one, ROM5, was able to cleave full-length MIC adhesins efficiently in a heterologous cell-based assay, and cleavage was inhibited by DCI, which is known to inhibit MPP1 and block invasion (Conseil et al. 1999; Carruthers et al. 2000). This enzyme localizes to the posterior surface of the parasite, the expected site of MPP1 activity (Fig. 7; Brossier et al. 2005; Dowse et al. 2005). Thus, TgROM5 is most likely the enzyme responsible for adhesin cleavage at the final stages of invasion, although genetic analysis is required to verify whether ROM5 is indeed MPP1 in Toxoplasma.

Surprisingly, the Plasmodium genome encodes eight distinct rhomboid-like genes, but not a ROM5 homolog, and several families of Plasmodium adhesins contain aromatic transmembrane residues that are not conducive for rhomboid cleavage. A recent analysis discovered that at least one adhesin is shed from invading bloodstage parasites by intramembrane cleavage, while an enzymatic analysis implicated two Plasmodium rhomboid enzymes with different substrate specificities that together fulfill the role of TgROM5 (Baker et al. 2006; O'Donnell et al. 2006). Moreover, all known families of Plasmodium adhesins can be cleaved by either or both Plasmodium rhomboid enzymes (Baker et al. 2006), raising the exciting possibility that rhomboid intramembrane cleavage may be the convergence point of several distinct invasion pathways at all stages of the malaria life cycle. Importantly, whether inhibiting rhomboid activity would block parasite invasion has not yet been explored, and to date no specific inhibitors of rhomboid function have been reported.

\section{Concluding perspectives: evolution of a signaling mechanism}

The presence of rhomboid proteins in all branches of life implies that they are unusually useful factors that originated early in the evolution of life and have been conserved. However, neither the reason for their implied significance nor the path of their evolution are understood. The key to answering these questions is rooted in understanding not only the sequence distribution of these genes, but more importantly, their functions across evolution.

From an evolutionary point of view, it is intriguing to note that the predominant function of rhomboid proteins is in regulating cell-to-cell communication in organisms as diverse as bacteria and animals. During parasite invasion of host cells rhomboid enzymes are cleaving type I membrane proteins with signaling and adhesion modules present on their extracellular side to dissolve adhesin-receptor junctions at the end of the invasion program. This is, at least in superficial terms, a similarity to their function in signaling, where cleavage of transmembrane precursors releases active signaling 
molecules. Whether signaling regulators have indeed been co-opted to fulfill this nonsignaling role, or whether this is a coincidence, remains open to debate. It should be noted that both Toxoplasma and Plasmodium encode numerous rhomboid enzymes, some of which could also be involved in parasite signaling and development.

The function of PARL/Pcp1 represents a case where a rhomboid's role clearly lies outside cell-cell communication. Sequence analysis indicates that PARL is more similar to prokaryotic than to eukaryotic rhomboid proteins, and could have been acquired from the invading bacterium that became the mitochondrial endosymbiont. Thus, the bacterial rhomboid protein, now encoded in the nucleus, could also have acquired a new role in mitochondrial membrane dynamics within a cell.

Currently there are hundreds of known rhomboid proteins, but we only know their functions in a small and biased sampling of about a dozen cases. Thus, it is not yet possible to ascertain whether their roles are predominantly as key regulators of cell signaling, or whether they fulfill a very broad spectrum of cellular functions. Sequence analysis indicates that rhomboid protein evolution is complex, as expected for such a widespread family of membrane proteins. Indeed, it is also possible that, contrary to the traditional view, rhomboid proteins originated in bacteria and spread by horizontal gene transfer (Koonin et al. 2003). Despite our limited knowledge, it is tempting to speculate that there is something versatile about this intramembraneous mode of regulation and the enzymes that catalyze it, and learning more about their functions could reveal insights into the evolution of regulatory mechanisms.

\section{Acknowledgments}

I am grateful to Rosanna Baker for expert assistance with drawing the illustrations. Research in the Urban laboratory is supported by grants from the National Institutes of Heath, and by a Career Award in the Biomedical Sciences from the BurroughsWellcome Fund (to S.U.).

\section{References}

Aikawa, M., Miller, L.H., Johnson, J., and Rabbege, J. 1978. Erythrocyte entry by malarial parasites. A moving junction between erythrocyte and parasite. J. Cell Biol. 77: 72-82.

Alexander, C., Votruba, M., Pesch, U.E., Thiselton, D.L., Mayer, S., Moore, A., Rodriguez, M., Kellner, U., Leo-Kottler, B., Auburger, G., et al. 2000. OPA1, encoding a dynamin-related GTPase, is mutated in autosomal dominant optic atrophy linked to chromosome 3q28. Nat. Genet. 26: 211-215.

Amutha, B., Gordon, D.M., Gu, Y., and Pain, D. 2004. A novel role of Mgm1p, a dynamin-related GTPase, in ATP synthase assembly and cristae formation/maintenance. Biochem. I. 381: 19-23.

Baker, R.P., Wijetilaka, R., and Urban, S. 2006. Two Plasmodium rhomboid proteases preferentially cleave different adhesins implicated in all invasive stages of malaria. PLOS Pathog. 2: e113.

Bang, A.G. and Kintner, C. 2000. Rhomboid and Star facilitate presentation and processing of the Drosophila TGF- $\alpha$ homolog Spitz. Genes \& Dev. 14: 177-186.

Bier, E., Jan, L.Y., and Jan, Y.N. 1990. rhomboid, a gene required for dorsoventral axis establishment and peripheral nervous system development in Drosophila melanogaster. Genes \& Dev. 4: 190-203.

Brossier, F., Jewett, T.J., Sibley, L.D., and Urban, S. 2005. A spatially localized rhomboid protease cleaves cell surface adhesins essential for invasion by Toxoplasma. Proc. Natl. Acad. Sci. 102: 4146-4151.

Brown, M.S., Ye, J., Rawson, R.B., and Goldstein, J.L. 2000. Regulated intramembrane proteolysis: A control mechanism conserved from bacteria to humans. Cell 100: 391-398.

Carruthers, V.B. 2002. Host cell invasion by the opportunistic pathogen Toxoplasma gondii. Acta Trop. 81: 111-122.

Carruthers, V.B., Giddings, O.K., and Sibley, L.D. 1999. Secretion of micronemal proteins is associated with Toxoplasma invasion of host cells. Cell. Microbiol. 1: 225-236.

Carruthers, V.B., Sherman, G.D., and Sibley, L.D. 2000. The Toxoplasma adhesive protein MIC2 is proteolytically processed at multiple sites by two parasite-derived proteases. J. Biol. Chem. 275: 14346-14353.

Cipolat, S., Rudka, T., Hartmann, D., Costa, V., Serneels, L., Craessaerts, K., Metzger, K., Frezza, C., Annaert, W., D'Adamio, L., et al. 2006. Mitochondrial rhomboid PARL regulates cytochrome $\mathrm{c}$ release during apoptosis via OPA1dependent cristae remodeling. Cell 126: 163-175.

Conseil, V., Soete, M., and Dubremetz, J.F. 1999. Serine protease inhibitors block invasion of host cells by Toxoplasma gondii. Antimicrob. Agents Chemother. 43: 1358-1361.

Delettre, C., Lenaers, G., Griffoin, J.M., Gigarel, N., Lorenzo, C., Belenguer, P., Pelloquin, L., Grosgeorge, J., Turc-Carel, C., Perret, E., et al. 2000. Nuclear gene OPA1, encoding a mitochondrial dynamin-related protein, is mutated in dominant optic atrophy. Nat. Genet. 26: 207-210.

Dimmer, K.S., Fritz, S., Fuchs, F., Messerschmitt, M., Weinbach, N., Neupert, W., and Westermann, B. 2002. Genetic basis of mitochondrial function and morphology in Saccharomyces cerevisiae. Mol. Biol. Cell 13: 847-853.

Dowse, T.J. and Soldati, D. 2005. Rhomboid-like proteins in Apicomplexa: Phylogeny and nomenclature. Trends Parasitol. 21: 254-258.

Dowse, T.J., Pascall, J.C., Brown, K.D., and Soldati, D. 2005. Apicomplexan rhomboids have a potential role in microneme protein cleavage during host cell invasion. Int. J. Parasitol. 35: 747-756.

Dutt, A., Canevascini, S., Froehli-Hoier, E., and Hajnal, A. 2004. EGF signal propagation during C. elegans vulval development mediated by ROM-1 rhomboid. PLOS Biol. 2: e334.

Dvorak, J.A., Miller, L.H., Whitehouse, W.C., and Shiroishi, T. 1975. Invasion of erythrocytes by malaria merozoites. Science 187: 748-750.

Edbauer, D., Winkler, E., Regula, J.T., Pesold, B., Steiner, H., and Haass, C. 2003. Reconstitution of $\gamma$-secretase activity. Nat. Cell Biol. 5: 486-488.

Esser, K., Tursun, B., Ingenhoven, M., Michaelis, G., and Pratje, E. 2002. A novel two-step mechanism for removal of a mitochondrial signal sequence involves the mAAA complex and the putative rhomboid protease Pcp1. J. Mol. Biol. 323: 835-843.

Freeman, M., Kimmel, B.E., and Rubin, G.M. 1992. Identifying targets of the rough homeobox gene of Drosophila: Evidence that rhomboid functions in eye development. Development 116: 335-346.

Frezza, C., Cipolat, S., Martins de Brito, O., Micaroni, M., Beznoussenko, G.V., Rudka, T., Bartoli, D., Polishuck, R.S., Danial, N.N., De Strooper, B., et al. 2006. OPA1 controls apoptotic cristae remodeling independently from mitochondrial fusion. Cell 126: 177-189. 
Gabay, L., Seger, R., and Shilo, B.-Z. 1997. In situ activation pattern of Drosophila EGF receptor pathway during development. Science 277: 1103-1106.

Gallio, M. and Kylsten, P. 2000. Providencia may help find a function for a novel, widespread protein family. Curr. Biol. 10: R693-R694.

Gallio, M., Sturgill, G., Rather, P., and Kylsten, P. 2002. A conserved mechanism for extracellular signaling in eukaryotes and prokaryotes. Proc. Nat1. Acad. Sci. 99: 12208-12213.

Gallio, M., Englund, C., Kylsten, P., and Samakovlis, C. 2004. Rhomboid 3 orchestrates Slit-independent repulsion of tracheal branches at the CNS midline. Development 131: 3605-3614.

Gerhart, J. 1999. 1998 Warkany lecture: Signaling pathways in development. Teratology 60: 226-239.

Glushakova, S., Yin, D., Li, T., and Zimmerberg, J. 2005. Membrane transformation during malaria parasite release from human red blood cells. Curr. Biol. 15: 1645-1650.

Golembo, M., Raz, E., and Shilo, B.Z. 1996. The Drosophila embryonic midline is the site of Spitz processing, and in duces activation of the EGF receptor in the ventral ectoderm. Development 122: 3363-3370.

Greenwood, B. and Mutabingwa, T. 2002. Malaria in 2002. Nature 415: 670-672.

Griparic, L., van der Wel, N.N., Orozco, I.J., Peters, P.J., and van der Bliek, A.M. 2004. Loss of the intermembrane space protein Mgm1/OPAl induces swelling and localized constrictions along the lengths of mitochondria. J. Biol. Chem. 279: 18792-18798.

Guichard, A., Biehs, B., Sturtevant, M.A., Wickline, L., Chacko, J., Howard, K., and Bier, E. 1999. Rhomboid and Star interact synergistically to promote EGFR/MAPK signaling during Drosophila wing vein development. Development 126: 2663-2676.

Guichard, A., Roark, M., Ronshaugen, M., and Bier, E. 2000. Brother of rhomboid, a rhomboid-related gene expressed during early drosophila oogenesis, promotes EGF-R/MAPK signaling. Dev. Biol. 226: 255-266.

Harari, P.M. 2004. Epidermal growth factor receptor inhibition strategies in oncology. Endocr. Relat. Cancer 11: 689-708.

Hartley, B.S. 1960. Proteolytic enzymes. Annu. Rev. Biochem. 29: 45.

Herlan, M., Vogel, F., Bornhovd, C., Neupert, W., and Reichert, A.S. 2003. Processing of Mgm1 by the rhomboid-type protease Pcp1 is required for maintenance of mitochondrial morphology and of mitochondrial DNA. J. Biol. Chem. 278: 27781-27788.

Herlan, M., Bornhovd, C., Hell, K., Neupert, W., and Reichert, A.S. 2004. Alternative topogenesis of Mgm1 and mitochondrial morphology depend on ATP and a functional import motor. J. Cell Biol. 165: 167-173.

Huynh, M.H., Barenau, K.E., Harper, J.M., Beatty, W.L., Sibley, L.D., and Carruthers, V.B. 2003. Rapid invasion of host cells by Toxoplasma requires secretion of the MIC2-M2AP adhesive protein complex. EMBO J. 22: 2082-2090.

Jaszai, J. and Brand, M. 2002. Cloning and expression of Ventrhoid, a novel vertebrate homologue of the Drosophila EGF pathway gene rhomboid. Mech. Dev. 113: 73-77.

Jewett, T.J. and Sibley, L.D. 2003. Aldolase forms a bridge between cell surface adhesins and the actin cytoskeleton in apicomplexan parasites. Mol. Cell 11: 885-894.

Jurgens, G., Wieschaus, E., Nusslein-Volhard, C., and Kluding, H. 1984. Mutations affecting the pattern of the larval cuticle in Drosophila melanogaster. Rouxs Arch. Dev. Biol. 193: 267-282.

Kanaoka, M.M., Urban, S., Freeman, M., and Okada, K. 2005.
An Arabidopsis rhomboid homolog is an intramembrane protease in plants. FEBS Lett. 579: 5723-5728.

Kappe, S., Bruderer, T., Gantt, S., Fujioka, H., Nussenzweig, V., and Menard, R. 1999. Conservation of a gliding motility and cell invasion machinery in Apicomplexan parasites. J. Cell Biol. 147: 937-944.

Kimberly, W.T., LaVoie, M.J., Ostaszewski, B.L., Ye, W., Wolfe, M.S., and Selkoe, D.J. 2003. $\gamma$-Secretase is a membrane protein complex comprised of presenilin, nicastrin, Aph-1, and Pen-2. Proc. Natl. Acad. Sci. 100: 6382-6387.

Kolodkin, A.L., Pickup, A.T., Lin, D.M., Goodman, C.S., and Banerjee, U. 1994. Characterization of Star and its interactions with sevenless and EGF receptor during photoreceptor cell development in Drosophila. Development 120: 17311745.

Koonin, E.V., Makarova, K.S., Rogozin, I.B., Davidovic, L., Letellier, M.C., and Pellegrini, L. 2003. The rhomboids: A nearly ubiquitous family of intramembrane serine proteases that probably evolved by multiple ancient horizontal gene transfers. Genome Biol. 4: R19.

Lee, J.R., Urban, S., Garvey, C.F., and Freeman, M. 2001. Regulated intracellular ligand transport and proteolysis control EGF signal activation in Drosophila. Cell 107: 161-171.

Lee, Y.J., Jeong, S.Y., Karbowski, M., Smith, C.L., and Youle, R.J. 2004. Roles of the mammalian mitochondrial fission and fusion mediators Fis1, Drp1, and Opa1 in apoptosis. Mol. Biol. Cell 15: 5001-5011.

Lemberg, M.K., Menendez, J., Misik, A., Garcia, M., Koth, C.M., and Freeman, M. 2005. Mechanism of intramembrane proteolysis investigated with purified rhomboid proteases. EMBO J. 24: 464-472.

Lohi, O., Urban, S., and Freeman, M. 2004. Diverse substrate recognition mechanisms for rhomboids; thrombomodulin is cleaved by mammalian rhomboids. Curr. Biol. 14: 236 241.

Lopez-Otin, C. and Overall, C.M. 2002. Protease degradomics: A new challenge for proteomics. Nat. Rev. Mol. Cell Biol. 3: 509-519.

Luetteke, N.C., Qiu, T.H., Peiffer, R.L., Oliver, P., Smithies, O., and Lee, D.C. 1993. TGF $\alpha$ deficiency results in hair follicle and eye abnormalities in targeted and waved-1 mice. Cell 73: 263-278.

Maegawa, S., Ito, K., and Akiyama, Y. 2005. Proteolytic action of GlpG, a rhomboid protease in the Escherichia coli cytoplasmic membrane. Biochemistry 44: 13543-13552.

Mann, G.B., Fowler, K.J., Gabriel, A., Nice, E.C., Williams, R.L., and Dunn, A.R. 1993. Mice with a null mutation of the TGF $\alpha$ gene have abnormal skin architecture, wavy hair, and curly whiskers and often develop corneal inflammation. Cell 73: 249-261.

Mayer, U. and Nusslein-Volhard, C. 1988. A group of genes required for pattern formation in the ventral ectoderm of the Drosophila embryo. Genes \& Dev. 2: 1496-1511.

McQuibban, G.A., Saurya, S., and Freeman, M. 2003. Mitochondrial membrane remodelling regulated by a conserved rhomboid protease. Nature 423: 537-541.

McQuibban, G.A., Lee, J.R., Zheng, L., Juusola, M., and Freeman, M. 2006. Normal mitochondrial dynamics requires rhomboid-7 and affects Drosophila lifespan and neuronal function. Curr. Biol. 16: 982-989.

Mesak, L.R., Mesak, F.M., and Dahl, M.K. 2004. Expression of a novel gene, gluP, is essential for normal Bacillus subtilis cell division and contributes to glucose export. BMC Microbiol. 4: 13 .

Meyerowitz, E.M. 2002. Plants compared to animals: The broadest comparative study of development. Science 295: 
1482-1485.

Nakagawa, T., Guichard, A., Castro, C.P., Xiao, Y., Rizen, M., Zhang, H.Z., Hu, D., Bang, A., Helms, J., Bier, E., et al. 2005. Characterization of a human rhomboid homolog, p100hRho/ RHBDF1, which interacts with TGF- $\alpha$ family ligands. Dev. Dyn. 233: 1315-1331.

Nusslein-Volhard, C., Wieschaus, E., and Kluding, H. 1984. Mutations affecting the pattern of the larval cuticle in Drosophila melanogaster: I. Zygotic loci on the second chromosome. Rouxs Arch. Dev. Biol. 193: 267-282.

O'Donnell, R.A., Hackett, F., Howell, S.A., Treeck, M., Struck, N., Krnajski, Z., Withers-Martinez, C., Gilberger, T.W., and Blackman, M.J. 2006. Intramembrane proteolysis mediates shedding of a key adhesin during erythrocyte invasion by the malaria parasite. J. Cell Biol. 174: 1023-1033.

Olichon, A., Baricault, L., Gas, N., Guillou, E., Valette, A., Belenguer, P., and Lenaers, G. 2003. Loss of OPA1 perturbates the mitochondrial inner membrane structure and integrity, leading to cytochrome c release and apoptosis. J. Biol. Chem. 278: $7743-7746$

Opitz, C., Di Cristina, M., Reiss, M., Ruppert, T., Crisanti, A., and Soldati, D. 2002. Intramembrane cleavage of microneme proteins at the surface of the apicomplexan parasite Toxoplasma gondii. EMBO J. 21: 1577-1585.

Pascall, J.C. and Brown, K.D. 2004. Intramembrane cleavage of ephrinB3 by the human rhomboid family protease, RHBDL2. Biochem. Biophys. Res. Commun. 317: 244-252.

Pascall, J.C., Luck, J.E., and Brown, K.D. 2002. Expression in mammalian cell cultures reveals interdependent, but distinct, functions for Star and Rhomboid proteins in the processing of the Drosophila transforming-growth-factor- $\alpha$ homologue Spitz. Biochem. J. 363: 347-352.

Pellegrini, L., Passer, B., Canelles, M., and Lefterov, I. 2001. PAMP and PARL, two novel putative metallopreoteases interacting with the $\mathrm{COOH}$-terminus of Presenilin-1 and 2. J. Alzheimers Dis. 3: 137-145.

Peschon, J.J., Slack, J.L., Reddy, P., Stocking, K.L., Sunnarborg, S.W., Lee, D.C., Russell, W.E., Castner, B.J., Johnson, R.S., Fitzner, J.N., et al. 1998. An essential role for ectodomain shedding in mammalian development. Science 282: 12811284.

Price, J.V., Clifford, R.J., and Schüpbach, T. 1989. The maternal ventralizing locus torpedo is allelic to faint little ball, an embryonic lethal, and encodes the Drosophila EGF receptor homolog. Cell 56: 1085-1092.

Rather, P.N. and Orosz, E. 1994. Characterization of aarA, a pleiotrophic negative regulator of the 2'-N-acetyltransferase in Providencia stuartii. J. Bacteriol. 176: 5140-5144.

Rather, P.N., Parojcic, M.M., and Paradise, M.R. 1997. An extracellular factor regulating expression of the chromosomal aminoglycoside 2'-N-acetyltransferase of Providencia stuartii. Antimicrob. Agents Chemother. 41: 1749-1754.

Rather, P.N., Ding, X., Baca-DeLancey, R.R., and Siddiqui, S. 1999. Providencia stuartii genes activated by cell-to-cell signaling and identification of a gene required for production or activity of an extracellular factor. J. Bacteriol. 181: 71857191.

Rawson, R.B., Zelenski, N.G., Nijhawan, D., Ye, J., Sakai, J., Hasan, M.T., Chang, T.Y., Brown, M.S., and Goldstein, J.L. 1997. Complementation cloning of S2P, a gene encoding a putative metalloprotease required for intramembrane cleavage of SREBPs. Mol. Cell 1: 47-57.

Roth, S., Neuman-Silberberg, F.S., Barcelo, G., and Schüpbach, T. 1995. Cornichon and the EGF receptor signaling process are necessary for both anterior-posterior and dorsal-ventral pattern formation in Drosophila. Cell 81: 967-978.
Rutledge, B.J., Zhang, K., Bier, E., Jan, Y.N., and Perrimon, N. 1992. The Drosophila spitz gene encodes a putative EGF-like growth factor involved in dorsal-ventral axis formation and neurogenesis. Genes \& Dev. 6: 1503-1517.

Sahin, U., Weskamp, G., Kelly, K., Zhou, H.M., Higashiyama, S., Peschon, J., Hartmann, D., Saftig, P., and Blobel, C.P. 2004. Distinct roles for ADAM10 and ADAM17 in ectodomain shedding of six EGFR ligands. J. Cell Biol. 164: 769779 .

Schejter, E.D. and Shilo, B.-Z. 1989. The Drosophila EGF receptor homolog (DER) gene is allelic to faint little ball, a locus essential for embryonic development. Cell 56: 1093-1104.

Scholz, H., Deatrick, J., Klaes, A., and Klambt, C. 1993. Genetic dissection of pointed, a Drosophila gene encoding two ETSrelated proteins. Genetics 135: 455-468.

Schulz, C., Wood, C.G., Jones, D.L., Tazuke, S.I., and Fuller, M.T. 2002. Signaling from germ cells mediated by the rhomboid homolog stet organizes encapsulation by somatic support cells. Development 129: 4523-4534.

Sesaki, H., Southard, S.M., Hobbs, A.E., and Jensen, R.E. 2003a Cells lacking Pcplp/Ugo2p, a rhomboid-like protease required for Mgmlp processing, lose mtDNA and mitochondrial structure in a Dnmlp-dependent manner, but remain competent for mitochondrial fusion. Biochem. Biophys. Res. Commun. 308: 276-283

Sesaki, H., Southard, S.M., Yaffe, M.P., and Jensen, R.E. 2003b. Mgm1p, a dynamin-related GTPase, is essential for fusion of the mitochondrial outer membrane. Mol. Biol. Cell 14: 2342-2356.

Sik, A., Passer, B.J., Koonin, E.V., and Pellegrini, L. 2004. Selfregulated cleavage of the mitochondrial intramembranecleaving protease PARL yields $\mathrm{P} \beta$, a nuclear-targeted peptide. J. Biol. Chem. 279: 15323-15329.

Soldati, D., Foth, B.J., and Cowman, A.F. 2004. Molecular and functional aspects of parasite invasion. Trends Parasitol. 20: 567-574.

Steiner, H. and Haass, C. 2001. Nuclear signaling: A common function of presenilin substrates? J. Mol. Neurosci. 17: 193198.

Steinmetz, L.M., Scharfe, C., Deutschbauer, A.M., Mokranjac, D., Herman, Z.S., Jones, T., Chu, A.M., Giaever, G., Prokisch, H., Oefner, P.J., et al. 2002. Systematic screen for human disease genes in yeast. Nat. Genet. 31: 400-404.

Sturtevant, M.A., Roark, M., and Bier, E. 1993. The Drosophila rhomboid gene mediates the localized formation of wing veins and interacts genetically with components of the EGF-R signaling pathway. Genes \& Dev. 7: 961-973.

Thomas, J.B., Crews, S.T., and Goodman, C.S. 1988. Molecular genetics of the single-minded locus: A gene involved in the development of the Drosophila nervous system. Cell 52: 133-141.

Tsruya, R., Schlesinger, A., Reich, A., Gabay, L., Sapir, A., and Shilo, B.Z. 2002. Intracellular trafficking by Star regulates cleavage of the Drosophila EGF receptor ligand Spitz. Genes \& Dev. 16: 222-234.

Urban, S. and Freeman, M. 2002. Intramembrane proteolysis controls diverse signalling pathways throughout evolution. Curr. Opin. Genet. Dev. 12: 512-518.

Urban, S. and Freeman, M. 2003. Substrate specificity of rhomboid intramembrane proteases is governed by helix-breaking residues in the substrate transmembrane domain. Mol. Cell 11: $1425-1434$.

Urban, S. and Wolfe, M.S. 2005. Reconstitution of intramembrane proteolysis in vitro reveals that pure rhomboid is sufficient for catalysis and specificity. Proc. Natl. Acad. Sci. 102: $1883-1888$. 
Urban, S., Lee, J.R., and Freeman, M. 2001. Drosophila rhomboid-1 defines a family of putative intramembrane serine proteases. Cell 107: 173-182.

Urban, S., Lee, J.R., and Freeman, M. 2002a. A family of Rhomboid intramembrane proteases activates all membranetether EGF ligands in Drosophila. EMBO J. 21: 42774286.

Urban, S., Schlieper, D., and Freeman, M. 2002b. Conservation of intramembrane proteolytic activity and substrate specificity in eukaryotic and prokaryotic Rhomboids. Curr. Biol. 12: $1507-1512$.

Urban, S., Brown, G., and Freeman, M. 2004. EGF receptor signaling protects smooth-cuticle cells from apoptosis during Drosophila ventral epidermis development. Development 131: 1835-1845.

Walder, K., Kerr-Bayles, L., Civitarese, A., Jowett, J., Curran, J., Elliott, K., Trevaskis, J., Bishara, N., Zimmet, P., Mandarino, L., et al. 2005. The mitochondrial rhomboid protease PSARL is a new candidate gene for type 2 diabetes. Diabetologia 48: 459-468.

Wang, Y., Zhang, Y., and Ha, Y. 2006. Crystal structure of a rhomboid family intramembrane protease. Nature Epub Oct. 11, 2006; doi: 10.1038/nature05255.

Wasserman, J.D. and Freeman, M. 1998. An autoregulatory cascade of EGF receptor signalling patterns the Drosophila egg. Cell 95: 355-364.

Wasserman, J.D., Urban, S., and Freeman, M. 2000. A family of rhomboid-like genes: Drosophila rhomboid-1 and roughoid/ rhomboid-3 cooperate to activate EGF receptor signalling. Genes \& Dev. 14: 1651-1663.

Waters, C.M. and Bassler, B.L. 2005. Quorum sensing: Cell-tocell communication in bacteria. Annu. Rev. Cell Dev. Biol. 21: 319-346.

Weihofen, A. and Martoglio, B. 2003. Intramembrane-cleaving proteases: Controlled liberation of proteins and bioactive peptides. Trends Cell Biol. 13: 71-78.

Weihofen, A., Binns, K., Lemberg, M.K., Ashman, K., and Martoglio, B. 2002. Identification of signal peptide paptidase, a presenilin-type aspartic protease. Science 296: 2215-2218.

Wilkinson, D.G. 2001. Multiple roles of EPH receptors and ephrins in neural development. Nat. Rev. Neurosci. 2: 155-164.

Wolfe, M.S. and Kopan, R. 2004. Intramembrane proteolysis: Theme and variations. Science 305: 1119-1123.

Wolfe, M.S., Xia, W., Ostaszewski, B.L., Diehl, T.S., Kimberly, W.T., and Selkoe, D.J. 1999. Two transmembrane aspartates in presenilin-1 required for presenilin endoproteolysis and $\gamma$-secretase activity. Nature 398: 513-517.

Wolpert, L. 1998. Principles of development. Oxford University Press, Oxford.

Wong, E.D., Wagner, J.A., Scott, S.V., Okreglak, V., Holewinske, T.J., Cassidy-Stone, A., and Nunnari, J. 2003. The intramitochondrial dynamin-related GTPase, Mgmlp, is a component of a protein complex that mediates mitochondrial fusion. J. Cell Biol. 160: 303-311.

Zhou, X.W., Blackman, M.J., Howell, S.A., and Carruthers, V.B. 2004. Proteomic analysis of cleavage events reveals a dynamic two-step mechanism for proteolysis of a key parasite adhesive complex. Mol. Cell. Proteomics 3: 565-576.

zür Lage, P., Jan, Y.N., and Jarman, A.P. 1997. Requirement for EGF receptor signalling in neural recruitment during formation of Drosophila chordotonal sense organ clusters. Curr. Biol. 7: 166-175. 


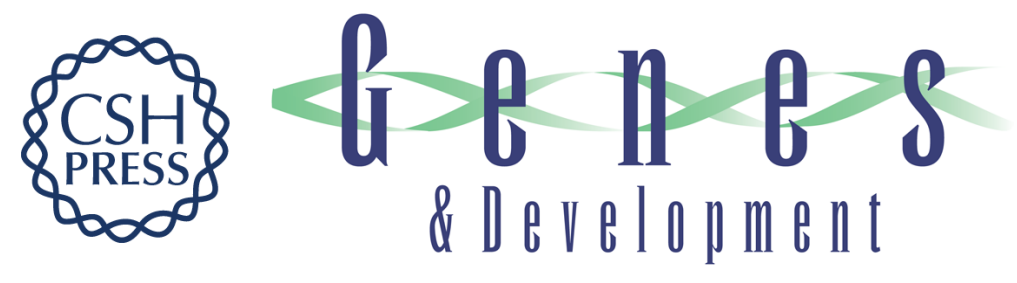

\section{Rhomboid proteins: conserved membrane proteases with divergent biological functions}

Sinisa Urban

Genes Dev. 2006, 20:

Access the most recent version at doi:10.1101/gad.1488606

References This article cites 108 articles, 49 of which can be accessed free at:

http://genesdev.cshlp.org/content/20/22/3054.full.html\#ref-list-1

License

Email Alerting Receive free email alerts when new articles cite this article - sign up in the box at the top Service right corner of the article or click here.

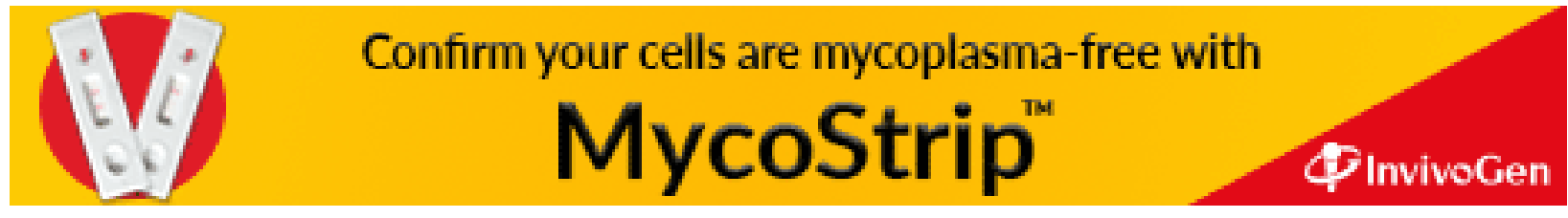

